\title{
Riemann Spaces of Class Two and Their Algebraic Characterization.
}

Part II.

Makoto Matsumoto.

(Received June 15, 1949)

In this paper we give a necessary and sufficient condition that a Riemann space $R_{n}(n \geq 8)$ be of class two, making use of the type number discussed in a preceding paper ${ }^{(1)}$.

\section{$\S$ 1. A reality condition}

Suppose that a Riemann space $R_{n}(n \geqq 6)$ of class two is of type $\geqq 3$, and put

$$
K_{i j}=p_{i j}+e q_{i j} \quad\left(e^{2}=-1\right) ;
$$

where the tensor $K_{i j}$ is the solution of $(1 \cdot 9)$ of the part I, i. e.

$$
M_{i j k l}=K_{i(j} K_{k l)},
$$

and $p$ 's and $q$ 's are all real. The tensor $M_{i j k l}$ in $(1 \cdot 2)$ is defined by $(1 \cdot 10)$ of the part I, i. e.

$$
M_{i j k l}=\frac{1}{2}\left(R_{c \cdot i(j}^{a} R_{|x| \cdot k l)}^{c}\right)
$$

Substituting $(1 \cdot 1)$ in $(1 \cdot 2)$ and equating to zero the imaginary parts we have

$$
p_{i(j} q_{k l)}+q_{i(j} p_{k l)}=0 \text {. }
$$

(A) Suppose that det. $|q| \neq 0$. Contracting (1.4) by $q^{k l}$ we have

$$
(n-4) p_{i j}+q^{a b} p_{a b} q_{i j}=0,
$$

and contracting it by $q^{i j}$ we have $q^{a b} p_{a b}=0$ for $n>2$. Therefore all of $p_{i j}$ are zero for $n \geq 6$. Hence the $K^{\prime}$ 's are pure imaginary except zero.

(B) Suppose that det. $|q|=0$. If the rank of $\|q\|$ is $2 \sigma(n>2 \sigma \geqq 6)$, we have similarly $p_{i j}=0$ for $i, j=1, \ldots \ldots, 2 \sigma$.

Next putting $k, l=1, \ldots \ldots, 2 \sigma$ and $i, j>2 \sigma$ in $(1 \cdot 4)$ we have $q_{k l} p_{i j}=0$, 
and therefore $p_{i j}=0$ for $i, j>2 \sigma$ if $2 \sigma \geqq 2$.

Finally putting $i, j, k=1, \ldots \ldots 2 \sigma$ and $l>2 \sigma$ in (1.4) we have

$$
p_{i l} q_{j k}+p_{k l} q_{i j}+p_{l j} q_{i k}=0
$$

Contracting this by $q^{i k}$ we have $p_{i l}=0$ for $i=1, \ldots \ldots 2 \sigma$ and $l>2 \sigma$ if $2 \sigma \geq 4$. Hence the following cases may occur:

$$
\begin{aligned}
& \text { (I) } 2 \sigma \geqq 6 ; \quad \text { then } p_{i j}=0 \quad(i, j=1, \ldots \ldots, n), \\
& \text { (II) } 2 \sigma=4 ; \text { then } p_{i j}=0 \quad(i=1, \ldots . ., n ; j=5, \ldots \ldots n) ; \\
& \text { (III) } 2 \sigma=2 ; \text { then } p_{i j}=0 \quad(i, j=3, \ldots \ldots n), \\
& \text { (IV) } 2 \sigma=0 ; \text { then } p_{i j}=0 \quad(i, j=1, \ldots \ldots n) .
\end{aligned}
$$

If the cases (II) or (III) are assumed to hold, we have immediately that a maximum value of rank of $\|K\|$ is four in contradiction to hypothesis on the type number and we have the

Lemma :......If a Riemann space $R_{n}(n \geqq 6)$ of class two is of type $\geqq 3$, the solutions $K$ 's of (1.2) are all real or pure imaginary except zero.

Now we put

$$
K_{(h, i, j, k, l, m)}^{2}=\left|\begin{array}{cccccc}
0 & K_{h i} & K_{h j} & K_{h k} & K_{h l} & K_{h m} \\
-K_{h i} & 0 & K_{i j} & K_{i k} & K_{i l} & K_{j m} \\
-K_{h l} & K_{i j} & 0 & K_{j k} & K_{j l} & K_{j m} \\
-K_{h l} & -K_{i k} & -K_{j k} & 0 & K_{k l} & K_{k m} \\
-K_{h l} & -K_{i l} & -K_{j l} & -K_{k l} & 0 & K_{l m} \\
-K_{h m} & -K_{i m} & -K_{j m} & -K_{k m} & -K_{l m} & 0
\end{array}\right|
$$

and

$$
M^{2}{ }_{(h, i, j, k, l m)}=\left|\begin{array}{cccccc}
0 & M_{j k l m} & M_{i k l m} & M_{i j l m} & M_{i j k m} & M_{i j k l} \\
-M_{j k l m} & 0 & M_{h k l m} & M_{h j l m} & M_{h j k m} & M_{l j k l} \\
-M_{i k l m}-M_{h k l m} & 0 & M_{h i l m} & M_{h i k m} & M_{h i k l} \\
-M_{i j l m} & -M_{h j l m} & -M_{h i l m} & 0 & M_{h i j m} & M_{h i j l} \\
-M_{i j k m}-M_{h j k m} & -M_{h i k m} & -M_{h i j m} & 0 & M_{h i j k} \\
-M_{i j k l} & -M_{h j k l} & -M_{h i k l} & -M_{h i j l} & -M_{h i j k} & 0
\end{array}\right|
$$

and let $K_{(h, i, j, k, l, m)}$ and $M_{(h, i, j k, l, m)}$ be respectively the Pfaff's aggregate of $K^{2}{ }_{(h, i, j, k, l, m)}$ and $M^{2}{ }_{(h, i, j, k, l, m)}$. 
From the theory ${ }^{(2)}$ of determinant, for example, let $\bar{K}_{h i}$ be the algebraic complement of $K_{h i}$ in det. $K_{(h, i, j, k, l, m)}$. The algebraic complement $K_{h i}$ in $K_{(h i, j, k, l, m)}$ is $M_{j k l m}$ as it is seen from $(1 \cdot 2)$. Then we have

$$
\bar{K}_{h i}=M_{j k l m} K_{(h, i, j, k, l, m)},
$$

and so on. Let $\bar{K}_{(h, i, j, k, l, n)}^{2}$ be the determinant, whose elements are $\bar{K}_{h i}, \ldots$, $\ldots, \bar{K}_{l m}$, then we have

$$
\bar{K}_{(h, i, j, k, l, m)}^{2}=M^{2}{ }_{(h, i, j, k, l, m)}\left\{K^{2}{ }_{(h, i, j, k, l, l, m)}\right\}^{3} .
$$

As $K_{(h, i, j, k, l, m)}^{2}$ is of order six, we have

$$
\bar{K}_{(h, i, j, k, l, m)}^{2}=\left\{K_{(h, i, j, k, l, m)}^{2}\right\}^{5},
$$

Now, from the hypothesis on the type number, we can take $h, i, j, k, l$, $m$ so that $K_{(h, i, j, k, l, m)}^{2}$ is not zero. Hence we have from (1.8)

$$
\left\{K_{(h, i, j, k, l, m)}^{2}\right\}^{2}=M_{(h, i, j, k, l, m)}^{2}
$$

$K^{2}{ }_{(h, i, j, k, l, m)}$ is positive, because it is square of real polynomial of $K_{h i}, \ldots \ldots, K_{l m}$. On the other hand we have from $(1 \cdot 2)$

$$
K_{(h, i, j, k, l, m)}^{2}=M T_{(h, i, j, k, l, m)} \text {. }
$$

Then we have

$$
M_{(h, i, j, k, l, m)} \geq 0
$$

If $K^{\prime} s$ be pure imaginary except zero and the rank of $\|K\|$ be $\geqq 6$, there is one of $K^{2}{ }_{(h, i, j, k, l, l, m)}$ which is negative. Hence from $(1 \cdot 10),(1 \cdot 11)$ and lemma we have the

Theorem I. I...... If a real Riemaun space $R_{n}(n \geqq 6)$ of class two is of type' $\geq 3$, solutions $K^{\prime}$ 's shall be real if, and only if, the inequality (1.11) is satisfied.

\section{$\S 2$. The resultant system}

From (1.9) we have the

Lemma : ......If a real Ricmann space $R_{n}(n \geqq 6)$ of class two is of type $\geqq 3$, it is necessary that 


$$
\sum M_{(h, i, j, k, l, m)}^{2}>0 ;
$$

where the summation is to be extended oucr all possible values of the indices appearing in the above determinant.

Further necessary conditions in the form of a system of linear homogeneous equations can be derived as follows. Let us write $(1.2)$ in their homogeneous form namely,

$$
A^{2} M_{i j k l}=K_{i(j} K_{k l)} .
$$

We multiply $(1.2)$ by $K_{h m}$ and subtract the expression obtained by interchanging $m$ and $l$ and then we have by means of (1.2)

$$
K_{i j} M_{h m l l}+K_{j k} M_{h m i l}+K_{k i} M_{h m j l}+K_{h m} M_{i j l k}+K_{m l} M_{i j h k}+K_{l h} M_{i j m k}=0 \text {. }
$$

Consider the equations $(2 \cdot 2)$ and $(2 \cdot 2)$ as a system for the determination of the unknowns $A$ and $K^{\prime}$ s. Since $R_{n}$ is of class two and of type $\geqq 3$, the system must admit such a solution that the matrix $\|K\|$ has rank $\geq 6$, hence the system composed of $(2 \cdot 2)$ and $(2 \cdot 3)$ must admit a non-trivial solution $\left(A, K^{\prime} s\right)$. Now we know from the theory ${ }^{(3)}$ of a system of homogeneous algebraic equations that the above equations $(2 \cdot 2)$ and (2.3) must admit a resultant system, i. e. a set of polynomials in the components $M$ 's such that the vanishing of these polynomials is necessary and sufficient for the existence of a non-trivial solution. Representing the resultant system of $(2 \cdot 2)$ and $(2 \cdot 3)$ by $R(M)$, it follows that

$$
R(M)=0
$$

is a necessary condition for a Riemann space $R_{n}$ to be of class two.

Suppose $(2 \cdot 4)$ to be satisfied. Let $\left(A, K^{\prime} s\right)$ be a ron-trivial solution of $(2 \cdot 2)$ and $(2 \cdot 3)$. Suppose $A=0$ in this solution. Then by the similar way as in $\S 1$ of the part I we have the rank of matrix $\|K\|^{\prime}$ to be zero or two. If the rank of $\|K\|$ is two, taking the coordinate system such that all of $K_{i j}$ are zero except $K_{12}$ and putting $i=1, j=2$ and $k, k, l$, $m=3, \ldots \ldots, n$ in $(2.3)$ we have $M_{h k l m}=0$ for $h, k, l, m=3 \ldots \ldots, n$. Next putting $i=h=1, j=2$ and $k, l, m=3, \ldots \ldots, n$ in $(2.3)$ we have $M_{1 k l m}=0$ for $k, l, m=3, \ldots \ldots, n$ and similarly $M_{2 k l m}=0$. Hence all of $M_{i j k l}=0$ except $M_{12 i j}$ in contradiction to $(2 \cdot 1)$ and it follows that all of $K^{\prime} s$ are zero in contradiction to the hypothesis. We must therefore have $A \neq 0$ so that the quantities $K_{i j} / A$ can be defined and these constitute a solution 
of $(1 \cdot 2)$. We thus have from the theorem $2 \cdot 1$ of the part I the

Theorem 2. I...... If à real Riemann space $R_{n}(n \geq 6)$ is of type $\geqq 3$ and the left-hand members of $(1 \cdot 2)$ are defined by $(1 \cdot 3)$, then the equations $(1 \cdot 2)$ will have solutions $K$ 's which are unique to within algebraic sign if, and only if, the inequality (2.1) and the equations (2.4) are satisfied. .

When conditions $(1 \cdot 11)$ are likewise imposed, it follows from the theorem 1.1 that the above solutions $K^{\prime} s$ will be real. In this case the polynomial inequality $(2 \cdot 1)$ can be replaced by the polynomial inequality

$$
\sum M_{(h, i, j, k, l, m)}>0
$$

of lower degree, the summation in this inequality and in $(2 \cdot 1)$ having the same significance. Hence we have the

Theorem 2. 2..... If a real Riemann space $R_{n}(n \geqq 6)$ is of type $\geq 3$ and the left-hand members of $(1 \cdot 2)$ are defined by $(1 \cdot 3)$, then the equation $(1.2)$ zeill have a real solutions $K^{\prime}$ 's which are unique to within algebraic sign if, and only if, the inequalities (1.12) and (2.5) and the equation (2.4) are satisfied.

Moreover we shall derive the explicit expression for ' $K$ 's. From the theory ${ }^{(4)}$ of determinant, we have

$$
\left(K_{h i}\right)^{2}\left\{K_{(h, i, j, k, l, m)}^{2}\right\}^{3}=\left|\begin{array}{cccc}
0 & \bar{K}_{j k} & \bar{K}_{j l} & \bar{K}_{j m} \\
-\bar{K}_{j l} & 0 & \bar{K}_{k l} & \bar{K}_{k m} \\
-\bar{K}_{j l} & -\bar{K}_{k l} & 0 & \bar{K}_{l m} \\
-\bar{K}_{j m} & -\bar{K}_{k m} & -\bar{K}_{l m} & 0
\end{array}\right|
$$

and from $(1 \cdot 7)$

$$
=\left\{K_{(h, i, j, k, l, m)}^{2}\right\}^{2}\left|\begin{array}{cccc}
0 & M_{h i l m} & M_{h i k m} & M_{h i k l} \\
-M_{h i l m} & 0 & M_{h i j m} & M_{h i j l} \\
-M_{h i k m} & -M_{h i j m} & 0 & M_{h i j k} \\
-M_{h i k l} & -M_{h i j l} & -M_{h i j k} & 0
\end{array}\right|
$$

Hence, let $M_{(h, i)}$ be the above determinant and we have

$$
\left(K_{h i}\right)^{2} M_{(h, i, j, k, l, m)}=M_{(h, i)} .
$$

If we hope to obtain the full expression of $K^{\prime} s$, we may discuss in 
similar way as in $\S 9$ in the Thomas's paper for Riemann spaces of class one. ${ }^{(6)}$

\section{§ 3. Tensor $\boldsymbol{E}_{i j k l}$}

Let a Riemann space $R_{n}(n \geqq 6)$ of class two be of type $\geqq 3$. We put

$$
E_{i j k l}^{\cdot}=H_{i, j}^{P} H_{k l}^{P} \quad(P=I, I l ; i, j, k, l=1, \ldots \ldots, n),
$$

and shall find the intrinsic expressions of $E^{\prime} s$. For this purpose, we shall first find the intrinsic expressions of $L$ 's defined by $(1 \cdot 1)$ in the part I, i. e.

$$
L_{a i b j}=H_{a i}^{I} H_{b j}^{I I}-H_{a j}^{I} H_{b i}^{I I}-H_{a i}^{I I} H_{b j}^{I}+H_{a j}^{I I} H_{b i}^{I} .
$$

(A) Suppose that det. $|K| \neq 0$ and contract (1.7) of the part I, i. e.

$$
N_{a b i j k l}=L_{a b b(j} K_{k l)}+K_{t(j} L_{|a| k \mid b i l)}
$$

by $K^{k l}$. As $L_{\text {aibj }}$ is skew-symmetric in $i$ and $j$, we have

$$
(n-4) L_{a i b j}+K^{k l} L_{a k b l} K_{s j}=K^{k l} N_{a b i j k l}
$$

Contracting $(3 \cdot 4)$ by $K^{i j}$ we have

$$
K^{i j} L_{a i b j}=\frac{1}{2(n-2)} K^{i j} K^{k l} N_{a b i j k l,}
$$

hence from $(3 \cdot 4)$ we have

$$
\begin{array}{r}
L_{a t b j}=\frac{1}{n-4} K^{c d} N_{a b i j c d}-\frac{1}{2(n-2)(n-4)} K_{i j} K^{c d} K^{f o} N_{a b c d f g} \\
(a, b, c, d, i, j=1, \ldots, n) .
\end{array}
$$

(B) Suppose that the rank of $\|K\|=2 \tau(n>2 \tau \geqq 6)$, and take $i, j$, $k, l=1, \ldots \ldots, 2 \tau$ and $a, b=1, \ldots \ldots, n$ in $(3 \cdot 3)$, and then we have similarly $\left(3 \cdot 5^{\prime}\right)$ obtained by taking $i, j, c, d, f, g=1, \ldots, \ldots, 2 \tau$ and $a, b=1, \ldots \ldots, n$ and $n=2 \tau$ in (3.5). Next taking $j>2 \tau ; i, k, l=1, \ldots, \ldots, 2 \tau$ and $a, b=1$, $\ldots . ., n$ in $(3 \cdot 3)$ we obtain

$$
L_{a t h j}=\frac{1}{2(\tau-1)} K^{c d} N_{a b i j c d}(j>2 \tau ; i, c, d=1, \ldots . ., 2 \tau ; a, b=1, \ldots . ., n)
$$


Finally taking $i, j>2 \tau ; k, l=1 \ldots . ., 2 \tau$ and $a, b=1, \ldots \ldots, n$ in $(3 \cdot 3)$ we have similarly

$$
L_{a t b j}=\frac{1}{2 \tau} K^{c d} N_{a b i j c d}(i, j>2 \tau ; c, d=1, \ldots \ldots 2 \tau ; a, b=1, \ldots \ldots n) .
$$

Hence we have uniquely the intrinsic expressions of $L$ 's from $(3 \cdot 3)$, if the conditions of the theorem 2.1 are satisfied.

Now, if we put

$$
S_{a i b j}=H_{a i}^{I} H_{b j}^{I I}-H_{a i}^{I I} H_{b j}^{I}(a, b, i, j=1, \ldots \ldots, n),
$$

and then we have from $(3 \cdot 2)$

$$
S_{a i b j}=\frac{1}{2}\left(L_{a i b j}+L_{i a j b}\right) \text {. }
$$

Next multiplying $(1 \cdot 6)$ of the part I, i. e.

$$
H_{a(i}^{Q} K_{|Q| \cdot j k)}^{P}=H_{c(i,}^{P} R_{|a| \cdot j k)}^{c},
$$

for $P=I I$ by $H_{b l}^{I}$ and for $P=I$ by $H_{b l}^{I I}$ and subtracting, we have from $(3 \cdot 1)$ and $(3 \cdot 8)$

$$
E_{a(i|b l|} K_{j k)}=S_{c(i|b| b \mid} R_{|a| \cdot \cdot j k)}^{c}
$$

By the similar way as that of finding $L ' s$, when $|K| \neq 0$, we have

$$
E_{a i n l}=\frac{1}{n-2} K^{j k}\left(S_{c i b l} R_{a \cdot j k}^{c}+2 S_{c j b l} R_{a \bullet k i}^{c}\right),
$$

and when the rank of $\|K\|$ is $2 \tau(\mathrm{n}>2 \tau \geqq 6)$, we have $\left(3 \cdot 12^{\prime}\right)$ obtained by taking $i, j . k=1, \ldots . ., 2 \tau$, and $a, b, c, l=1, \ldots \ldots, n$ and $n=2 \tau$ in $(3 \cdot 12)$, and

$$
\begin{aligned}
E_{a i b l}=\frac{1}{2 \tau} K^{j k} & \left(S_{c i b l} R_{a \cdot j k}^{c}+2 S_{c j b l} R_{a \cdot k i}^{c}\right) \\
& (a, i, c, l=1, \ldots . ., n ; \jmath, k=1, \ldots . ., 2 \tau ; i>2 \tau) .
\end{aligned}
$$

On the other hand from $(3 \cdot 1)$ and $(3 \cdot 8)$, we have immediately

$$
E_{a i b j}=E_{b j a i}=E_{i a b j} \quad(a, b, i, j=1, \ldots \ldots n),
$$

and 


$$
S_{a b c l} S_{i j k l}=\left|\begin{array}{ll}
E_{u b l j} & E_{a b k l} \\
E_{c l l i j} & E_{c l l l l}
\end{array}\right|(a, b, c, d, i, j, k, l=1, \ldots \ldots, n) .
$$

Finally we have from the Gauss equation

$$
R_{i j k l}=E_{i k j l}-E_{i l j k}(i, j, k, l=1, \ldots \ldots, n) .
$$

\section{§ 4. The Gauss equation}

In the first place we shall pay attention to the following facts.

Remar. I. If we interchange the positive directions of normals $B_{P}^{\cdot \alpha}$ to a $n$-dimensional variety $S_{n}$ in a $(n+2)$-dimensional euclidean space - $E_{n+2}$, the algebraic sign of $H_{i j}^{P}$ changes according to $H_{i j}^{P}=B_{i, j}^{\cdot \alpha} B_{P}^{\cdot \alpha}$ :

Remark II. Let $B_{P}^{\cdot \alpha}$ and $\overline{B_{P}^{* \alpha}}$ be tivo systems of mutually orthogonal unit vectors normal to $S_{n}$ in $E_{n+2}$. With reference to $B_{P}^{\cdot \alpha}$ and $\bar{B}_{p}^{\cdot \alpha}$ we have respectively $H_{i j}^{P}$ and $\bar{H}_{i j}^{P}$. The formulae of transformation of normal systems are

$$
\overline{b_{P}^{\circ} \cdot \alpha}=l_{P}^{Q} B_{Q}^{\cdot \alpha} ;
$$

where $\|l\|$ is orthogonal matrix. We can deduce

$$
\bar{H}_{i j}^{P}=l_{P}^{Q} H_{i j}^{Q} \text {. }
$$

If we put, for example, $\bar{H}_{11}^{I I}=0$, i. e.

$$
l_{11}^{I} H_{11}^{I}+l_{11}^{I I} H_{11}^{I I}=0,
$$

from this equation $l_{P}^{Q}(P, Q=I, I I)$ are determined to within algebraic sign. Therefore, for example, the algebraic sign of

$$
\bar{H}_{11}^{P}=l_{1}^{I} H_{11}^{I}+l_{1}^{I I} H_{11}^{I I}
$$

can be selected arbitrarily and then other $\bar{H}_{i j}^{P}$ are all determined.

Now, if a real Riemann space $R_{n}(n \geqq 6)$ is of class two and of type $\geq 3$, it is necessary that $(1 \cdot 11),(2 \cdot 4)$ and $(2 \cdot 5)$ are satisfied. Then $K^{\prime} s$ is expressed intrinsically. Making use of $K$ 's, we have intrinsic expressions of $L ' s, S^{\prime} s$ and $E^{\prime} s$. Therefore $(3 \cdot 14),(3 \cdot 15)$ and $(3 \cdot 16)$ are also necessary conditions for $R_{n}$ to be of class two. These necessary conditions $(1 \cdot 11),(2 \cdot 4),(3 \cdot 5),(3 \cdot 14),(3 \cdot 15)$ and $(3 \cdot 16)$ are constracted 
only by $g_{i j}$ and their partial derivatives.

Conversely we shall prove the

Theorem 4. I :...... If a real Riemann space $R_{n}(n \geqq 6)$ is of type $\geq 3$, then there will be a set of functions $H_{i j}^{P}\left(=H_{j i}^{P}\right)(P=I, I I: i, j=1, \ldots$ $\ldots, n)$ satisfying the Gauss equation if, and only if, the inequalities (1.11) and $(2 \cdot 5)$, and the equations $(2 \cdot 4),(3 \cdot 14),(3 \cdot 15)$ and $(3 \cdot 16)$ are satisfied.

Taking $a=i, b=j, c=k, d=l$ in $(3 \cdot 15)$ we have

$$
E_{i j i j} E_{k l k l}-\left(E_{i j k l}\right)^{2}=\left(S_{i j k l}\right)^{2}
$$

Suppose that all of $E_{i j i j}=0$ and then all of $E_{i j k l}=0$ from $(4 \cdot 1)$, because $E$ 's and $S$ 's are real. Therefore from $(3 \cdot 16) R_{i j k l}$ is zero tensor in contradiction to the hypothesis on the type number. Now suppose, for example, $E_{1111} \neq 0$. We take $H_{11}^{I I}=0$ and

$$
H_{11}^{1}=\sqrt{E_{1111}}
$$

where algebraic sign is arbitrary (Cf. Re. II). Next we take

$$
E_{11 i j}=H_{11}^{I} H_{i j}^{I} \quad(i, j=1, \ldots \ldots, n),
$$

and then we have uniquely such a set of functions $H_{i j}^{l}(i, f=1, \ldots \ldots, n)$ that is symmetric in $i$ and $j$ from $(3 \cdot 14)$. Finally we take

$$
S_{11 t j}=H_{11}^{I} H_{i j}^{I I}(i, j=1, \ldots \ldots, n) \text {, }
$$

and then we have uniquely a set of functions $H_{i j}^{I I}(i, j=1, \ldots, \ldots, n)$ and it is symmetric in $i$ and $j$, because interchanging $a$ and $b$, or $c$ and $d$ in $(3 \cdot 15)$ we obtain

$$
S_{a b c d}=S_{b a c l l}=S_{a b l c} \text {. }
$$

Now we shall prove that those $H_{i j}^{P}$ satisfy $(3 \cdot 1)$. In fact, taking $a=b=i=j=1$ in $(3 \cdot 10)$ we have from $(4 \cdot 2),(4 \cdot 3)$ and $(4 \cdot 4)$

$$
\left|\begin{array}{ll}
\left(H_{11}^{I}\right)^{2} & H_{11}^{I} H_{j l}^{I} \\
H_{11}^{I} H_{c d}^{I} & E_{c d k l}
\end{array}\right|=\left(H_{11}^{I}\right)^{2} \cdot H_{k l}^{I} H_{c d}^{\eta},
$$

hence we can deduce $(3 \cdot 1)$ immediately. Finally from $(3 \cdot 16)$ those satisfy the Gauss equation and therefore we have the theorem 4.1.

If $H_{i j}^{P}$ are real, we have from $(3 \cdot 1) E_{i j i j} \geq 0(i, j=1, \ldots \ldots n)$ and so, from $(3 \cdot 15)$, matrix $\left\|E_{i j k l}\right\|(i, j:$ row $p, l:$, column $)$ is positive semi- 
definite. Conversely the matrix $\left\|E_{i j k l}\right\|$ is positive semi-definite, we can have real $H_{i j}^{p}$ by the above method, hence we have the

Theorem 4, 2 : ...... If a real Riemanin space $R_{n}(n \geqq 6)$ is of type $\geq 3$, then there will be a set of real functions $H_{i j}^{P}\left(=H_{j i}^{P}\right)(P=I, I I ; i, j$ $=1, \ldots \ldots, n)$ satisfying the Gauss equation if, and only if, the inequalitics $(1 \cdot 11)$ and $(2 \cdot 5)$, and the equations $(2 \cdot 4),(3 \cdot 14),(3 \cdot 15)$ and $(3 \cdot 16)$ are satisfied; and $\left\|E_{i j k l}\right\|$ is positive semi-definite.

We have from the theorem 2.4 of the part I and theorem 4.1 the

Theorem 4, 3: ..... If a real Riemann space $R_{n}(n \geq 8)$ is of type $\geqq 4$, then there will be two sets of functions $H_{i j}^{P}\left(=H_{j i}^{P}\right)$ and $H_{Q i}^{P}\left(=-H_{P i}^{P}\right)$ $(P, Q=I, I I ; i, j=\ldots \ldots, n)$ satiyfying the Gauss, Codazzi and Ricce equations if, and only if, the inequalities $(1 \cdot 11)$ and $(2 \cdot 5)$, and the equations $(2 \cdot 4),(3 \cdot 14),(3 \cdot 15)$ and $(3 \cdot 16)$ are satisfied; and the matrix $\left\|E_{i j k l}\right\|$ is positive semi-definite.

If $H_{i j}^{P}$ are real, $H_{Q i}^{P}(P, Q=I, I I ; \imath=1, \ldots \ldots, n)$ satisfying the Codazzi equaion are also real (Cf. Allendoerfer's paper), ${ }^{(6)}$ hence we have the most remarkable theorem:

Theorem 4. 4: ...... If a real Riemann space $R_{n}(n \geqq 8)$ is of type $\geqq 4$, then $R_{n}$ will be of class two if, and only if, the inequalities $(1 \cdot 11)$ and $(2 \cdot 5)$, and the equations $(2 \cdot 4),(3 \cdot 14),(3 \cdot 15)$ and $(3 \cdot 16)$ are satisficd; and the matrix $\left\|E_{i j k l}\right\|$ is positive semi-definite.

For remark, a solution $K^{\prime} s$ of $(1.2)$ is unique to within algebraic sign for type $\geqq 3$ and $g^{a b}\left(H_{a i}^{l 1} H_{b j}^{l}-H_{a j}^{l 1} H_{b i}^{I}\right)$ satisfy $(1.2)$ as the result of the Gauss equation, hence the equation (Cf. $\S .1$ of the part I)

$$
K_{i j}=g^{a b}\left(H_{a i}^{1 I} H_{b j}^{l}-H_{a j}^{I I} H_{b i}^{l}\right)
$$

are satisfied to within algebraic sign.

Revised Oct. 20, 1949

\section{References}

1) M. Matsumoto: Riemann spaces of class two and their algebraic characterization (part I), J. Math. Soc. Japan.

2) G. Kowalewski : Einführung in die Determinantententheorie, P. 142.

3) B. L. van der Waerden: Moderne Algebra 2, P. 14.

4) G. Kowalewski : 1. c. P. 80 .

5) Acta Math. 67 (1936).

6) Amer. J. Math. 61 (1939).

Mathematical Institute Doshisha University 